\title{
La vuelta de los sonidos casi a los 100 años. ¿Cuál va a ser el límite?
}

\section{The return of sounds at almost 100 years of age. Which will be the limit?}

\author{
Marcos Goycoolea V. ${ }^{1}$, Byanka Cagnacci B. ${ }^{2}$, Jorge Rufs B. ${ }^{3}$, Raquel Levy J. ${ }^{1}$, \\ Catherine Catenacci S. ${ }^{4}$, Maricarmen Andrade A. ${ }^{5}$, Jacqueline Scherpenisse I. ${ }^{6}$
}

\section{Resumen}

El implante coclear (IC) es el tratamiento estándar para las sorderas profundas en niños y adultos. En adultos mayores esta indicación ha pasado a ser cada día más común. Se describe un abordaje para implantes cocleares en adultos mayores usando como hilo conductor el caso clínico de una mujer de 98 años y 9 meses con hipoacusia neurosensorial severa que ya no se beneficiaba de sus audífonos. Las evaluaciones fueron conducidas por las áreas de otología, cardiología, neurología y anestesiología. Con las aprobaciones de las áreas médicas, la cirugía de implante coclear en el oído izquierdo fue realizada con anestesia local y sedación. No hubo complicaciones intra ni postoperatorias. La rápida recuperación permitió el alta al segundo día posoperatorio. El implante fue activado al mes de operada con todos los electrodos estando activos. Se lograron umbrales para tonos puros de $25 \mathrm{~dB}$ HL en campo libre y discriminación en silencio con IC en oído izquierdo y audífono en oído derecho para frases del 76\% y para palabras familiares del $100 \%$. El implante coclear fue una alternativa adecuada para esta paciente, posiblemente la implantada de mayor edad en el mundo, y debe ser considerado una alternativa razonable para el adulto mayor con sordera profunda. La decisión quirúrgica debe estar enfocada en las condiciones generales de salud más que en la edad cronológica.

Palabras clave: Implante coclear e hipoacusia en el adulto mayor, implante coclear en la persona mayor en el mundo, anestesia para el adulto mayor, edad e hipoacusia.

\footnotetext{
Abstract

Cochlear implant (IC) is the standard treatment for profound deafness in children and adults. In the elderly this indication is becoming more common every day. An approach to cochlear implants in the elderly is described, using as a common thread the case of a 98 years and 9 months old woman with severe bilateral gradually progressive sensorineural hearing loss who did not benefit from her hearing aids. She underwent comprehensive multispecialty medical evaluation including otolaryngology, neurology, cardiology and anesthesiology. She underwent cochlear implantation under local anesthesia and sedation. No intra or postoperative complications occurred. Recovery was quick and she was discharged on the second postoperative day. The device was activated at 1-month post-surgery and all electrodes were active. Free field thresholds for pure tones were $25 \mathrm{~dB} H \mathrm{H}$ and discrimination in silence with IC in left ear and hearing aid in right ear for sentences were $76 \%$ and for familiar words $100 \%$. Cochlear implantation resulted in an adequate alternative for this elderly patient, possibly the oldest implanted individual in the world. Cochlear implantation should be considered a reasonable alternative for elderly patients with profound hearing loss. The surgical decision should focus more on the general health conditions than on the chronological age.

Key words: Cochlear implantation, hearing loss in the elderly, cochlear implants in the elderly, oldest person with cochlear implant, anesthesia for the elderly, aging and hearing loss.
}

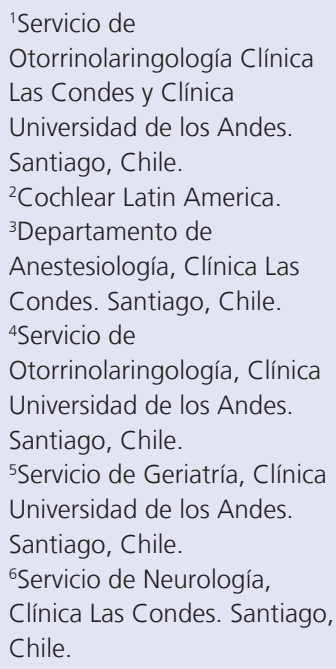

Los autores declaran no tener conflictos de interés.

Recibido el 22 de abril de 2020. Aceptado el 7 de octubre de 2020.

Correspondencia: Marcos Goycoolea V. Av. Paul Harris Oriente 11133 Las Condes. Santiago, Chile. Email: marcos.goycoolea@ gmail.com 


\section{Introducción}

El envejecimiento es un proceso fisiológico en el cual la estructura y capacidad funcional de los diferentes órganos se deterioran gradualmente, deterioro del cual no está exento el sentido de la audición. Un 30\% de las personas sobre 65 años y el 70\% de los mayores de 75 años tienen algún grado de hipoacusia neurosensorial ${ }^{1,2}$. Las expectativas de vida han cambiado. En el 2020 el porcentaje de adultos sobre 65 años son el 16\% de la población ${ }^{1,2}$.

Los efectos de la hipoacusia pueden ser devastadores y se pueden traducir en problemas de comunicación e integración y eventualmente en aislamiento, soledad, decaimiento cognitivo e incluso demencia ${ }^{3,4}$. En la mayoría de las personas con hipoacusia neurosensorial bastarán audífonos y un programa de rehabilitación, sin embargo, hay individuos para los cuales audífonos no son suficientes y requieren un implante coclear. Los implantes cocleares son actualmente el estándar de tratamiento para hipoacusias neurosensoriales severas a profundas en niños y adultos, y los adultos mayores son candidatos potenciales para este tratamiento ${ }^{5-7}$.
El objetivo de este relato es describir el caso clínico de una mujer de 98 años y 9 meses con hipoacusia neurosensorial severa que ya no se beneficiaba de sus audífonos y que fue operada de implante coclear.

\section{Caso Clínico}

M.R. es una mujer nacida en 1919, diagnosticada con hipoacusia simétrica hace 20 años, la cual fue de lenta progresión y para la cual usó audífonos desde entonces en forma satisfactoria. Su audición y discriminación se fueron deteriorando hasta que los audífonos le eran insuficientes para una comunicación adecuada. La paciente solicitó un implante coclear a fines de 2017, presentado las audiometrías descritas en la Figura 1.

\section{Antecedentes médicos}

Hipertensión arterial estable y uso de marcapasos cardíaco. La paciente es dueña de un predio agrícola que administra exitosamente hasta la fecha. Es muy activa socialmente. Al presentar a la paciente los riesgos involucrados en el procedimiento quirúrgico, ella fue

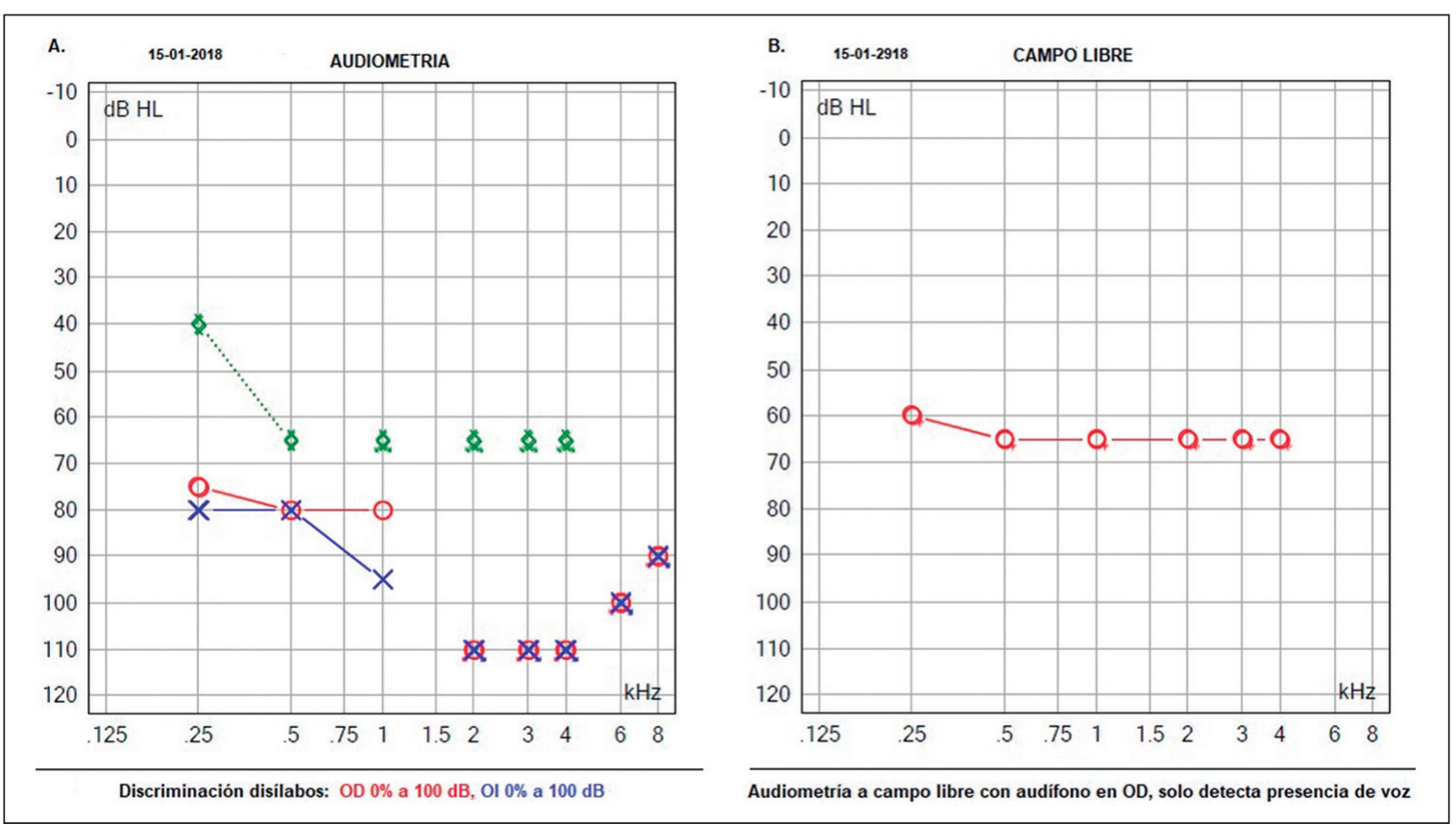

Figura 1. A. Audiometría preoperatoria. B. Audiometria a campo libre con audífono en oído derecho preoperatoria. 
enfática al decir que "prefería tomar el riesgo de morir que transformarse en una muerta viviente aislada del mundo". El examen neurológico no mostró defectos focales. Se le efectuó una evaluación cognitiva con minimental test abreviado para adulto mayor (MMSE-EFAM) y estaba normal (18 puntos). Posterior a las evaluaciones por los especialistas en cardiología y anestesiología, se aprobó en forma unánime el procedimiento, considerando su estado general de salud y sus condiciones neurocognitivas. Dada su edad se sugirió hacer el procedimiento con anestesia local y sedación.

La paciente fue implantada en el oído izquierdo en marzo de 2018. Se usó anestesia local (carbocaína al 2\% con epinefrina al 1 por $100.000)$ y sedación con propofol. El procedimiento se hizo en 2 horas y sin variaciones en la técnica quirúrgica habitual con anestesia general ${ }^{8}$. Se colocaron todos los electrodos in- tracocleares a través de la ventana redonda sin inconvenientes (Figura 2). El implante fue un Cochlear Nucleus CI24RE Nucleus Freedom Recto. El procedimiento fue bien tolerado por la paciente y sin complicaciones intra ni postoperatorias. Fue dada de alta al segundo día postoperatorio. La paciente se movilizaba sola no tenía dolor, náuseas ni vértigo.

A la fecha lleva dos años de operada y, durante este tiempo, cumplió los 100 años. Después de la activación del procesador y, gradualmente, la paciente nota una mejora en su audición, con una buena ganancia funcional como se presenta en la Figura 3. La paciente ha preferido el uso bimodal, es decir, implante a izquierda y audífono a derecha. Ha logrado un buen control de la intensidad de su voz y su comunicación en un ambiente tranquilo con uno o dos interlocutores, es óptima. Ha vuelto a "encontrarse" con sonidos importantes para

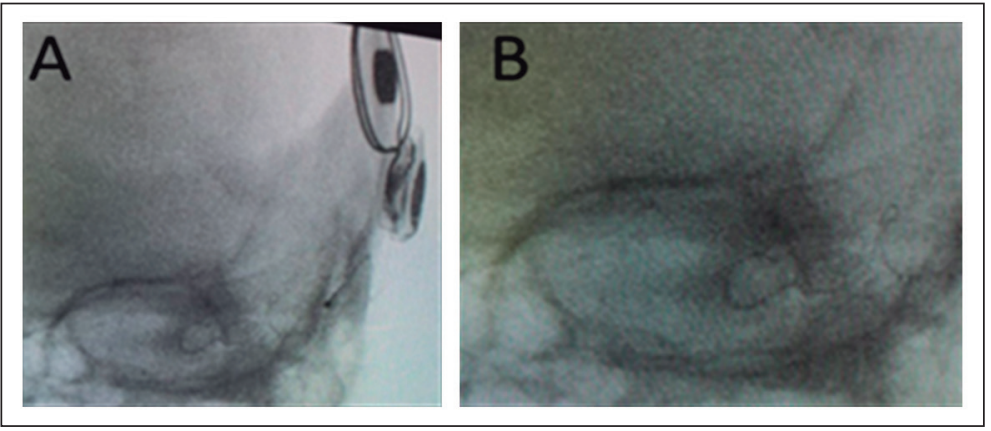

Figura 2. Implante coclear poscirugía.

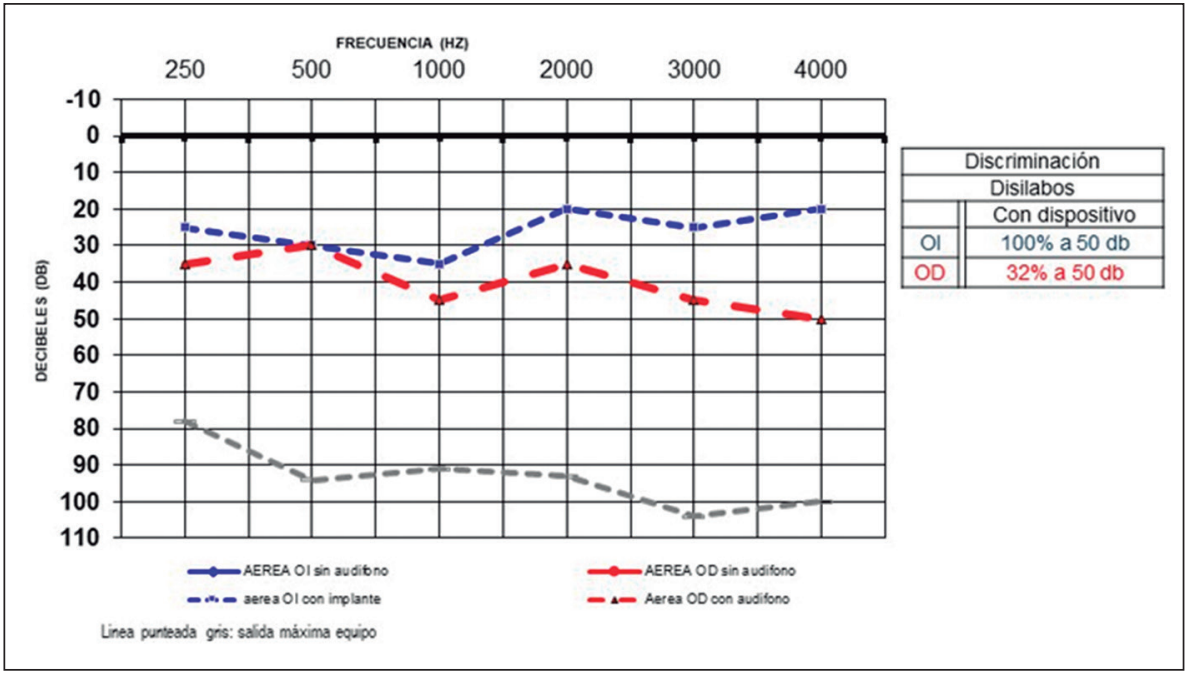

Figura 3. Audiometría a campo libre con audífono en OD e implante coclear en OI. 
ella como el ruido del mar. En la actualidad, es capaz de discriminar un $76 \%$ en frases y un $100 \%$ en palabras conocidas en ambiente silencioso sin apoyo de lectura labial. Esto le ha permitido estar conectada con su entorno y básicamente el seguir siendo la persona independiente que ha sido a lo largo de su vida.

\section{Discusión}

El envejecimiento es un proceso fisiológico en el cual la estructura y la capacidad funcional de los diferentes órganos se deteriora con el tiempo. Este proceso está influenciado por muchos factores, entre ellos estilo de vida, medio ambiente, genética, factores socioeconómicos y enfermedades ${ }^{2,9,10}$. En el adulto mayor estas discapacidades funcionales están compensadas en un delicado balance, sin embargo, cuando se requiere una respuesta fuera de la rutina o en situaciones de estrés como en cirugía, algunas de estas discapacidades se hacen manifiestas ${ }^{9,10}$.

Si bien en nuestras instituciones implantamos adultos mayores y actualmente tenemos seis pacientes implantados mayores de 85 años de edad, el caso de nuestra paciente de avanzada edad es excepcional. De acuerdo con una reciente revisión de la literatura ${ }^{11}$, esta paciente sería la persona más añosa que haya sido implantada en el mundo. En este caso, la buena salud y predisposición anímica de la paciente, sumado al apoyo familiar fueron factores importantes en nuestra decisión quirúrgica. A pesar de que la cirugía convencional de implante coclear con anestesia general es segura para los pacientes añosos, siempre está presente la discusión en relación con los riesgos de la anestesia general. Está descrito que los adultos mayores pueden ser operados sin riesgos o dolor indebido bajo anestesia local y sedación ${ }^{12}$, y nuestra publicación lo confirma en un caso de implante coclear. Los adultos mayores también tienen más riesgos de complicaciones posoperatorias como infecciones adquiridas, eventos tromboembólicos, deshidratación y menor consumo de nutrientes, los cuales pueden ser disminuidos restaurando la movilidad y la autonomía lo antes posible en el postoperatorio ${ }^{10}$.

Actualmente, el desafío anestésico no está limitado al intraoperatorio, sino que se extiende al período perioperatorio. La complicación más temida es el deterioro cognitivo postoperatorio, el cual está asociado a diferentes frecuencias en diferentes tipos de cirugías ${ }^{10,13}$. Se reconocen varios factores de riesgo relacionados con la aparición de deterioro cognitivo postoperatorio. Estos pueden ser intrínsecos y extrínsecos. Los intrínsecos son aquellos propios de cada paciente, siendo los más importantes la edad, deterioro cognitivo previo, capacidad funcional disminuida, bajo nivel educacional, abuso de medicamentos y "fragilidad". Los extrínsecos tienen que ver con las características de la cirugía, la magnitud y duración, la inflamación y el manejo fisiológico y de fármacos anestésicos durante la cirugía. Aparentemente el factor de riesgo más importante es la respuesta inflamatoria causada por la cirugía ${ }^{10}$. La profundidad anestésica ha sido asociada a una incidencia mayor de deterioro neurocognitivo y a ello se debe la recomendación de monitorización continua de ésta ${ }^{13}$. Monitoreo permanente es esencial para mantener la homeostasis fisiológica (eg. gases en sangre, electrolitos, salida urinaria, profundidad de la sedación y oxigenación cerebral ${ }^{13}$.

Basado en estos antecedentes, en nuestra paciente se organizó un procedimiento quirúrgico corto asociado a un proceso inflamatorio moderado y localizado, bajo anestesia local y propofol en infusión continua, los cuales permitieron mantener los parámetros fisiológicos estables.

La mejoría en la calidad de vida, en la ansiedad, en el aislamiento social, estrés, depresión y autoestima que los pacientes añosos experimentan después de un implante coclear es un hecho asumido por todos los autores $^{3,14,15}$. Aunque sean datos subjetivos, esto fue claramente observado en la paciente. La mejoría de la audición en pacientes mayores de 60 años resulta en una calidad de vida que es perdurable e independiente de la calidad de la audición ${ }^{15}$, lo que también ocurrió con esta paciente. Más aún, el foco actual está en la relación entre la hipoacusia, la demencia y el deterioro cognitivo ${ }^{3,5}$. Estudios recientes han sugerido un efecto positivo del implante coclear sobre el deterioro cognitivo ${ }^{5}$. Más aún, Mosnier y cols. ${ }^{5}$ mostraron que el rendimiento cognitivo promedio en pacientes implantados mejoraba a los 6 meses. De hecho, más allá 
de la edad, el foco debería estar en la edad biológica de nuestros pacientes en relación con sus condiciones generales más que en su edad cronológica. Por ello sugerimos que se realicen más estudios focalizados en pacientes "super añosos (> 85)" para aportar evidencias a estas sugerencias.

Finalmente, cabe mencionar que una hipoacusia neurosensorial bilateral severa a profunda poslocutiva, en Chile está cubierta por la Ley Ricarte Soto. Por lo tanto, adultos mayores que desarrollen estos grados de pérdida auditiva podrían potencialmente ser beneficiados por esta ley.

\section{Conclusión}

El implante coclear fue una alternativa adecuada para esta paciente -posiblemente la implantada de mayor edad en el mundo- y debe ser considerado una alternativa razonable para el adulto mayor con sordera profunda. La decisión quirúrgica para colocar un implante en un adulto mayor debe estar enfocada en las condiciones generales de salud más que en la edad cronológica.

\section{Bibliografía}

1. Goycoolea M. Introduction and General Perspective of sensorineural hearing loss. Rev Med Clin Condes. 2016;27(6):721-730.

2. Goycoolea M. Inner ear and understanding the message. En: Goycoolea MV, ed. The music of the spheres and the magic of hearing. Ciudad de Panamá: Jay Pee Highlights Medical Publishers 2017;55-89.

3. Lin FR, Yaffe K, Xia J, et al. Hearing loss and cognitive decline in older adults. JAMA Intern Med 2013;173:293-9.

4. Délano P. Hipoacusia. Un nuevo factor de riesgo para demencia. Rev Otorrinolaringol Cir Cabeza Cuello. 2017;77:237-238.

5. Mosnier I, Bebear JP, Marx M, et al. Improvement of cognitive function after cochlear implantation in elderly patients. JAMA Otolaryngol Head Neck Surg. 2015;141:442-50.

6. Yang Z, Cosetti M. Safety and outcomes of cochlear implantation in the elderly: a review of recent literature. J Otol. 2016;11:1-6.

7. Dietz A, Wüstefeld M, Niskanen M, Löppönen H. Cochlear Implant Surgery in the Elderly. Otol Neurotol. 2016;37(5):487-491.

8. Goycoolea MV, Ribalta GL. Exploratory tympanotomy: An integral Part of Cochlear Implantation. Acta Otolaryngol. 2003;123:223-226.

9. Brown E, Purdon P. Curr Opin Anaesthesiol. 2013;26(4):414-419.

10. Fu H, Fan L, Wang T. Perioperative neurocognition in elderly patients. Curr Opin Anaesthesiol. 2018;31(1):24-29.

11. Claes AJ, Van de Heyning P, Gilles A, Van Rompaey V, Mertens G. Cognitive outcomes after cochlear implantation in older adults: A systematic review. Cochlear Implants Int. 2018;19(5):239-254.

12. Strøm C. Challenges in anaesthesia for elderly. Singapore Dental Journal. 2014;35:23-29.

13. Guenther U. Patients prone for postoperative delirium: preoperative assessment, perioperative prophylaxis, postoperative treatment. Curr Opin Anaesthesiol. 2016; 29(3):384-390.

14. Olze H, Knopke S, Gräbel S, Szczepek AJ. Rapid positive influence of cochlear implantation on the quality of life in adults 70 years and older. Audiol Neurotol. 2016; 21(1):43-47.

15. Ramos-Macías Á, Falcón González JC, BorkoskiBarreiro SA, et al. Health-related quality of life in adult cochlear implant users: a descriptive observational study. Audiol Neurotol. 2016;21(1):3642. 